\title{
La politique du voile en France : droits et valeurs dans la fabrique de la laïcité
}

\section{Philippe Portier}

\section{(2) OpenEdition \\ 1 Journals}

Édition électronique

URL : http://journals.openedition.org/rdr/920

DOI : $10.4000 /$ rdr.920

ISSN : 2534-7462

Éditeur

Presses universitaires de Strasbourg

\section{Édition imprimée}

Date de publication : 8 novembre 2016

Pagination : 61-81

ISBN : 978-2-86820-959-7

ISSN : 2493-8637

\section{Référence électronique}

Philippe Portier, «La politique du voile en France : droits et valeurs dans la fabrique de la laïcité », Revue du droit des religions [En ligne], 2 | 2016, mis en ligne le 11 février 2020, consulté le 19 novembre 2020. URL : http://journals.openedition.org/rdr/920 ; DOI : https://doi.org/10.4000/rdr.920

\section{(c) (7) \&}

La revue du droit des religions est mise à disposition selon les termes de la Creative Commons Attribution - Pas d'Utilisation Commerciale 4.0 International - CC BY-NC 4.0. 


\section{LA POLITIOUE DU VOILE EN FRANCE : DROITS ET VALEURS DANS LA FABRIQUE DE LA LAḯITÉ}

\section{Philippe PORTIER}

EPHE / CNRS, Groupe Sociétés, Religions, Laïcités (GSRL)

\section{RÉSUMÉ}

La loi du 15 mars 2004 sur le port de signes religieux à l'école publique et celle du 11 octobre 2010 sur la dissimulation du visage dans l'espace public s'inscrivent dans une même perspective de substantialisation de la laïcité française. Appuyé sur l'analyse des idées et des intérêts des acteurs, cet article se propose de présenter la façon dont cette nouvelle normativité s'est construite.

\section{Abstract}

The Act of 15 March 2004 on conspicuous religious signs in public schools and the Act of 11 October 2010 prohibiting the concealment of the face in the public space are part of the same perspective of substantialisation of French laïcité. Based on the analysis of the ideas and of the interests of the involved actors, this article aims to present how this new normativity was elaborated. 
A u cours des années 1960-1970, la République française n'entretient qu'un 1 rapport lointain avec le fait musulman. Si elle accueille sur son sol des
populations venues des terres d'islam, c'est en les laissant, globalement, à leur auto-organisation sur le terrain religieux. Une sorte d'indifférence se manifeste en la matière, explicable en partie par le fait que la société d'alors valorise massivement la différence. On entre dans un autre schéma dans les années 1980. Au paradigme indifférentiste se substitue le paradigme interventionniste : une politique globale de l'islam se fait jour, articulée autour d'un vaste corpus de productions discursives, législatives, réglementaires, jurisprudentielles ${ }^{1}$.

Cette mutation politique a partie liée avec une mutation sociale. D'abord, la population musulmane s'est enracinée dans la société française. Elle est de passage jusqu'aux années 1970 : l'industrie avait fait venir d'Afrique des travailleurs solitaires, qui repartiraient au pays, la retraite venue. La politique de regroupement familial, développée à partir de la présidence de Valéry Giscard d'Estaing, change la donne. L'immigration est désormais d'installation: les travailleurs font souche, avec leur entourage, selon un processus que renforcent les règles du jus soli. Le moment est marqué aussi par l'accentuation de l'identité islamique. Les premiers installés rêvaient d'une citoyenneté ordinaire : ils étaient volontiers ancrés dans un éthos universaliste, ce dont témoignera encore la "marche pour l'égalité » en 1983. Le milieu des années 1980 voit s'imposer une pensée de la reconnaissance : alors que le modèle de l'intégration républicaine marque le pas, les générations nouvelles se laissent volontiers tenter par des revendications particularistes, ce dont témoignent en 1989 les voiles d'Épinal et de Creil, et, une décennie plus tard, la vogue du halal, dont le principe se déploie bientôt dans tous les domaines de l'existence ${ }^{2}$. Troisième changement : l'internationalisation de la question musulmane. À l'abri de ses frontières hier encore, l'Occident devient le réceptacle d'une violence que ses promoteurs relient à leur fidélité religieuse : la fatwa de Khomeiny contre Rushdie est le point de départ d'un processus que compléteront bientôt, avec le soutien d'une jeunesse formée en son sein même, les attentats meurtriers de Paris, Londres et Madrid, euxmêmes sous le surplomb du onze septembre.

Porté par une philosophie inédite de la cohésion nationale ${ }^{3}$, le gouvernement français a fait face aux défis de cette conjoncture en produisant une

1. LAURENCE J., The emancipation of Europe's Muslims. The State's role in minority integration, Princeton University Press, 2012.

2. Kepel G., Quatre-vingt-treize, Paris, Gallimard, 2012.

3. Donzelot J., « Refonder la cohésion sociale », Esprit, déc. 2006, p. 5-23. 
politique à trois volets. Premier registre : celui de la «citoyennisation ${ }^{4}$. Il s'est agi d'étendre aux populations nouvellement implantées les droits civils et sociaux de la population ordinaire. En dépit des résistances de la société, on a, dans ce cadre, non seulement favorisé la construction des mosquées, mais encore développé certaines politiques - sur un mode territorial et non ethnique - d'affirmative action sur le terrain économique (politique des zones franches) ou dans le domaine éducatif (politique des zones d'éducation prioritaire). Deuxième registre : celui de l'institutionnalisation. Son souci de les « intégrer» (selon le vocable en cours depuis les années 1990) a conduit l'État à favoriser la constitution d'interfaces avec les populations musulmanes, depuis le Conseil de réflexion sur l'islam de France institué par Pierre Joxe en 1990 jusqu'à l'Instance de dialogue avec l'islam pensée en 2015 par Manuel Valls, en passant par le Conseil français du culte musulman encouragé par Nicolas Sarkozy en 2003. Dernier registre : celui de l'acculturation. Le pouvoir politique s'est donné pour objectif, sans remettre en cause la nécessité de faire droit aux logiques de la diversité, de rassembler les populations musulmanes autour des «valeurs de la République ». En ce sens, il y a lieu de signaler la réinstallation, dans les programmes scolaires, des cours d'éducation morale et civique, abandonnés au tournant des années 1960-1970, mais aussi les politiques de proscription des signes religieux dans divers secteurs de la société française. Empressons-nous de souligner que jamais, bien sûr, les textes en question ne visent explicitement les musulmans : non seulement parce que, socialement, la nécessité de faire lien vaut pour bien d'autres catégories de la population, mais aussi, parce que, juridiquement, une telle logique donnerait lieu à une censure immédiate sous le chef d'atteinte au principe de non-discrimination.

Sur la question du port des signes religieux, deux lois majeures ont été adoptées au cours de ces dernières années. Celle du 15 mars 2004 sur le port de signes religieux à l'école publique ${ }^{5}$, celle du 11 octobre 2010 sur la dissimulation du visage dans l'espace public ${ }^{6}$, votées l'une et l'autre par une immense majorité de parlementaires. Comment les analyser ? Certains auteurs les ont saisies à partir du paradigme de la continuité. C'est le cas,

4. Lorcerie F., « À l'assaut de l'agenda public. La politisation du voile islamique en 2003-2004 », in La politisation du voile. L'affaire en France, en Europe et dans le monde arabe, Paris, L'Harmattan, 2005, p. 11.

5. Loi n ${ }^{\circ}$ 2004-228 du 15 mars 2004 encadrant, en application du principe de laïcité, le port de signes ou de tenues manifestant une appartenance religieuse dans les écoles, collèges et lycées publics.

6. Loi $\mathrm{n}^{\circ}$ 2010-1192 du 11 octobre 2010 interdisant la dissimulation du visage dans l'espace public. 
par exemple, de Martha Nussbaum qui a vu dans la production de ces deux lois une illustration de l'attachement pérenne de la France à une culture de la défiance, issue de la Révolution française, à l'égard des adhésions religieuses $^{7}$. D'autres se sont placés, au contraire, sous l'égide du paradigme de la dissociation. Ainsi en va-t-il, par exemple, de Patrick Weil. En tant qu'elle protège la liberté des mineures contre les pressions de leur entourage, la loi de 2004, explique-t-il, est une production libérale, inscrite du reste dans la ligne des lois laĩques de la Troisième République; ce n'est nullement le cas de la loi de 2010 qui, en restreignant l'autonomie des femmes majeures, accuse clairement son caractère illibéral ${ }^{8}$.

Ce texte voudrait dessiner une autre voie : il entend rappeler que les deux lois, rompant avec le modèle procéduraliste mis en place sous la Troisième République, s'inscrivent l'une et l'autre dans une même perspective de substantialisation de la laïcité française. Appuyée sur l'analyse des idées et des intérêts des acteurs, la démonstration adopte un mode séquentiel d'exposition, en présentant successivement les référentiels sur les fondements desquels s'est construite la politique française du voile (1), les instruments dont les acteurs politiques ont fait usage pour la faire triompher (2), les législations enfin sur lesquelles elle a débouché (3).

\section{LES RÉFÉRENTIELS}

Une politique publique est souvent explicable par l'action décisoire des « lanceurs d'alerte $»^{9}$. La loi de 2004 trouve son origine lointaine dans l'intervention en 1989 d'Ernest Chènière, principal du collège de Creil, où trois jeunes filles ont refusé de répondre à son injonction de retirer leur foulard dans l'établissement. La loi de 2010 dans celle, en 2009, d'André Gerin,

7. Nussbaum M., «Veiled threats?», The New York Times Opiniator, 11 juill. 2010 ; « Beyond the veil. A response ", Ibid., 15 juill. 2010. - Dans la même ligne, bien que l'ouvrage ait été publié avant l'affaire de la burqa, Bowen J., Why the French don't like headscarves? Islam, the State and public space, Princeton University Press, 2007.

8. WeIl P., «Voile et burqa en France. Deux lois d'interdiction aux sens différents», in ERHENFreund J. et Gisel P. (dir.), Religieux, société civile, politique, Lausanne, Antipodes, 2012, p. 83.

9. Chateaureynaud le définit ainsi : «Toute personne, groupe ou institution qui, percevant les signes précurseurs d'un danger ou d'un risque, interpelle une ou plusieurs puissances d'action, dans le but d'éviter un enchaînement catastrophique, avant qu'il ne soit trop tard. » Chateaureynaud F., "Lanceur d'alerte », in Casillo I. (dir.), Dictionnaire critique et interdisciplinaire de la participation, Paris, GIS Démocratie et Participation, 2013. 
député communiste de Vénissieux, qui, excédé par la montée en puissance du salafisme dans sa ville, porte l'idée de créer une mission parlementaire sur le port du voile intégral dans l'espace public. Il reste que ces lanceurs d'alerte ne peuvent faire prévaloir leur interprétation du problème qu'ils entendent résoudre que si leur appel trouve appui sur une "culture publique ${ }^{10}$ qui légitime leur action. Or, la culture publique évolue au cours des années 1990-2000. Elle était, depuis mai 1968, marquée par une intelligence singulariste du monde, faisant toute sa part au droit à la différence. On la voit alors se recomposer autour d'un modèle bien plus universaliste, où la diversité ne se trouve justifiée que dans l'articulation qui la subsume sous la logique du commun.

Même si d'autres foyers de sens, médiatiques et politico-administratifs, y ont évidemment contribué, cette évolution de l'imaginaire social - ce que la sociologie politique appelle le référentiel de l'action publique - est largement redevable aux élaborations de la sphère intellectuelle ${ }^{11}$. En son sein, deux tendances s'affrontent. D'un côté, les tenants d'une laïcité « inclusive », rassemblés autour, notamment, de Michel Wieviorka, qui a consacré un ouvrage central au concept de différence dans les années 1990, de Jean Baubérot dont les positions sur la laïcité se sont construites contre l'universalisme unificateur $^{12}$, ou encore de Joël Roman. De l'autre, les défenseurs d'une laïcité « cohésive », réunis autour, par exemple, d'Élisabeth Badinter, d'Alain Finkielkraut ou d'Henri Pena-Ruiz. Au cœur de leur débat, on trouve, en premier lieu, la question du statut du sujet. Les « inclusivistes » pensent la subjectivité sous la catégorie de l'identité, qu'ils relient au récit que chacun se donne pour apparaître au monde. Dans ce schéma, le voile ne peut être assimilé a priori à une abdication de sa propre dignité. Il peut être au contraire, face à des processus de relégation sociale ou d'oppression familiale, le moyen d'affirmer une singularité, de témoigner d'une agentivité, de conquérir une reconnaissance. Le sociologue Raphaël Liogier, qui appartient aussi à cette constellation pluraliste, résume parfaitement ce mode de penser :

« On pourrait croire que la résurgence de ce voile relève d'une imposition masculine, et/ou d'une poussée de radicalisme islamique. Or, ce qui

10. Gusfield J., La culture des problèmes publics. L'alcool au volant : la production d'un ordre symbolique, Paris, Économica, 2009.

11. LeYva K., «Multiculturalisme et laïcité en France : les trois républicanismes du rapport Stasi », Dialogue, n 54, 2016, p. 647-684.

12. Portier Ph., "Jean Baubérot et l'étude de la laïcité. Un tournant théorique ", in Zuber V., Cabanel P., Liogier R. (éds.), Croire, s'engager, chercher. Autour de Jean Baubérot, du protestantisme à la laïcité, Turnhout, Brepols, 2016. 
en prend l'apparence, et est perçu comme telles par les autres, n'est ni l'une ni l'autre pour ses protagonistes. Ce voile - spécifiquement celui qui se développe maintenant dans les sociétés industrielles avancées et non le voile musulman en général - est non seulement majoritairement volontaire mais hypervolontaire, si l'on peut dire : il traduit un désir d'ascétisme, de changement existentiel total, le désir d'une reconversion radicale, d'exhiber son identité, de la rendre visible. ${ }^{13}$

Les « assimilationnistes », qui ne distinguent guère, eux non plus, entre le voile simple et le voile intégral, tiennent un discours strictement opposé. Il n'est pas vrai, expliquent-ils, que le port du voile procède d'une liberté; il est le plus souvent imposé par les milieux familiaux et militants. L'analyse s'affirme dès 1989, au moment de l'affaire de Creil : "En autorisant de facto le foulard islamique, symbole de la soumission féminine, vous donnez un blanc-seing aux pères et aux frères, c'est-à-dire au patriarcat le plus dur de la planète. En dernier ressort, ce n'est plus le respect de l'égalité des sexes et du libre arbitre qui fait loi en France. $"{ }^{14}$ La chose est, à leurs yeux, d'autant plus insupportable qu'elle sépare l'individu du corps social - la fameuse exigence d' « adunation » chère à Sieyès - pour l'enfermer dans des communautés segmentées. Mais ce discours comporte aussi une ontologie de l'habit, qui trouve en son fond ce postulat que le sujet se définit d'abord, comme le voulaient les Lumières, par sa raison: le voile est, en soi, comme l'affirmait déjà Torné en 1792 à propos de la soutane des prêtres, un « monument d'oppression », qui signale un consentement à la domination, uniment masculine et religieuse. À rebours de ce que pensent les inclusivistes, on n'admet donc pas ici que le vêtement islamique puisse donner lieu à un jeu sémantique : sa signification n'est pas construite, mais donnée. On le lit dans cette déclaration d'Élisabeth Badinter devant la mission parlementaire présidée par André Gerin :

«J'observe qu'il existe de l'égalité des sexes deux approches opposées. L'une la nôtre, celle des démocraties, se résume en quatre mots : mêmes droits, mêmes devoirs. Ici la notion abstraite d'humanité l'emporte sur les différences biologiques, notamment sur la différence sexuelle. Puis il y a l'autre, celle des obscurantistes, celle aussi dont ont usé certains démocrates sincères, les naturalistes. Pour eux, [...] les sexes sont égaux dans leurs différences. ${ }^{15}$

13. Liogier R., "Le voile intégral comme trend hypermoderne », Multitudes, $\mathrm{n}^{\circ} 42,2010$, p. 16 .

14. Badinter É., Debray R. et al., « Foulard islamique. Profs, ne capitulons pas ! », Le Nouvel Observateur, 2 nov. 1989.

15. Rapport d'information sur la pratique du port du voile intégral sur le territoire national, Paris, Assemblée nationale, 26 janv. 2010, n 2262. 
La question du statut de l'État est un autre point de divergence. La tendance multiculturaliste se refuse, pour sa part, à souscrire à l'idée d'une privatisation des identités culturelles. De leur point de vue, cette privatisation est une négation de l'identité du sujet, mais aussi une consécration, non point de l'universel comme le veut le récit républicain, mais de la culture, très particulariste en fait, de la nation. Joël Roman l'écrit ainsi : «Libéral, le républicanisme français l'est dans la mesure où il valorise plus que tout l'autonomie de l'individu, en tant que celui-ci est censé obéir à la seule raison. Mais il n'est pas moins communautaire, puisqu'il fait de l'inscription de cet individu dans une singularité historique (l'exception française) et de sa prise en charge par l'État la condition de son émancipation. ${ }^{16}$ Certains analystes, tels Marwan Mohammed ou Nacira Guénif, ont pu ajouter que ces politiques assimilationnistes témoignaient d'un tropisme postcolonial. Comment penser alors la fonction politique? L'État doit se faire, non point le recteur des existences de ses assujettis, mais le scribe de leurs aspirations, sur le fondement de l'assomption de leurs droits subjectifs, notamment de leurs droits culturels. Neutre axiologiquement, il n'a nullement vocation à se placer au service d'une morale supérieure. Seul compte le choix de l'individu, pour peu sans doute qu'il s'opère librement, et qu'il ne porte pas atteinte, dans son déploiement pratique, aux libertés d'autrui non plus qu'à l'ordre public matériellement défini. On aurait tort, ajoutent les tenants du modèle inclusiviste, de voir dans cette politique de la reconnaissance un obstacle à l'intégration sociale. On admet d'autant plus le collectif qu'on se trouve admis à y faire valoir ses droits. Farhad Khosrokhavar l'explique ainsi :

«Plus le particularisme est banni du champ public et plus, par un effet de compensation, se "surpolitise" tout phénomène public. Ainsi une affaire comme celle des foulards se trouve-t-elle dotée d'un sens "politique" là où, compte tenu de ses dimensions et de son contenu, elle aurait dû faire l'objet de mesures et de négociations ad hoc et locales, à l'abri de toute politisation intempestive. $»^{17}$

Rien de cette conception purement procédurale du politique au sein du courant néo-républicain. On voit apparaître là un parti-pris en faveur d'une citoyenneté de la rectification : le pouvoir étatique se trouve requis de surélever ses administrés jusqu'à la sphère de la raison, en les arrachant

16. ROMAn J., « Un multiculturalisme à la française », Esprit, juin 1995, p. 153.

17. Khosrokhavar F., «L'universel abstrait, le politique et la construction de l'islamisme comme forme d'altérité », in Wieviorka M. (éd.), Une société fragmentée ?, Paris, La Découverte, 1997, p. 129. 
à l'ordre de leurs appartenances aliénantes. Cette intelligence du politique débouche sur une politique de l'interdit : le voile ne saurait avoir droit de cité. Dans les années 1990, on entend que l'interdit frappe le voile simple dans les écoles. Les années 2000 voient les assimilationnistes vouloir étendre la mesure à d'autres espaces, et notamment aux entreprises privées ou aux universités. Ils militent évidemment pour la prohibition totale du voile intégral. Les textes de justification sont légion en la matière. Retenons simplement celui-ci, d'Henri Pena-Ruiz. Son discours reprend l'argument de l'émancipation, non sans signaler les impasses du nationalisme sarkozyste :

« L'opposition du national et de l'étranger est nauséabonde. Cela veut dire qu'il n'y a pas à rejeter une tenue parce qu'“elle n'est pas de chez nous", mais parce qu'elle est incompatible avec le droit et la liberté des femmes. Si c'est le cas du voile intégral ou du voile partiel, ce n'est pas seulement en France qu'il n'est pas "bienvenu". C'est partout dans le monde. Nous avons un devoir de solidarité à l'égard des femmes qui, en Iran et partout dans le monde, refusent le voile. Il en va de nos responsabilités internationalistes. Que diront ces courageuses militantes si la République laïque à laquelle elles se réfèrent comme à un exemple consacre le port du voile qu'elles combattent ? ${ }^{18}$

L'argument de l'intégration est sollicité de même. Si l'État doit intervenir contre les prisons de tissu, c'est aussi pour renforcer, en rupture avec toutes les segmentations ou individualistes ou communautaristes qui le menacent, la cohésion du corps social :

«L'idée de bien commun à tous est au cœur de ma philosophie sociale. Mais il y a plusieurs façons d'être unis. Soit on s'unit en se soumettant. Soit on s'unit en s'émancipant. Comment concevoir l'être en commun ? Je soutiens une conception de la vie en commun des êtres singuliers en refusant que les particularités légitimes des groupes soient imposées à tous. [...] L'homme est un zoon politikon comme dit Aristote, c'est-àdire un "animal civique", un "animal citoyen", un être qui s'accomplit dans et par la cité. Il fallait donc remettre en valeur cette idée de communauté, contre le communautarisme. ${ }^{19}$

Les élites politiques se sont progressivement laissé convaincre par l'argumentaire néo-républicain. Sans doute n'en ont-elles pas repris la totalité des attendus (elles ont, en particulier, persisté à maintenir, quoique sous contrôle,

18. «Il n'est pas acceptable de sacrifier l'émancipation laïque à la libération sociale », Le Monde, 19 févr. 2010.

19. Propos recueillis par Charles CONTE : « Le cœur et la raison : entretien avec Henri PenaRuiz », Les idées en mouvement, $\mathrm{n}^{\circ}$ 221, sept.-oct. 2014. 
l'idée de diversité ${ }^{20}$ ). Elles ont cependant constitué leur référentiel d'action publique à partir du milieu des années 1990 sur le fondement d'une laïcité bien plus marquée par le principe de cohésion que dans la période antécédente. Jacques Chirac le déclare ainsi, lors de la cérémonie de réception du rapport Stasi, le 17 décembre 2003 : «Pilier de notre Constitution [...], [la laïcité requiert] l'intégration de tous dans le respect des différences. ${ }^{21}$ Cette conversion est le fruit sans doute du travail performatif de la production idéologique à l'instant aperçue, à laquelle ont contribué, par ailleurs, toute une série de rapports d'experts dans les années 1980-2000. Elle est le produit aussi de la mutation de la sphère politique : du fait de la consolidation du Front national, et de la droitisation de l'opinion publique, les partis de gouvernement - la droite dans un premier temps, la gauche bientôt - ont été amenés à cultiver une conception volontiers unificatrice du politique, elle-même appuyée sur une conception de plus en plus patrimonialiste du vivre ensemble. À ce tableau des causalités, il faudrait ajouter de surcroît le poids des mobilisations corporatives comme celles, pour le voile simple, des chefs d'établissement scolaire qui n'entendaient plus régler seuls la gestion des «dissidences musulmanes » au sein de leurs établissements.

\section{LES INSTRUMENTS}

Comment construire, à partir de ce système d'idées, un système de normes ? Sur le dossier qui nous occupe, on a assisté, au cours de la période, à une réévaluation de l'articulation des deux grands instruments de la régulation sociojuridique. Les années 1970 avaient été, en rupture avec le légicentrisme issu du rousseauisme culturel, le moment de l'affirmation de l'État de droit, construit autour de l'autonomisation de l'appareil constitutionnel ${ }^{22}$. L'affaire des voiles a fait resurgir le primat de l'État légal : c'est par la loi, et souvent en contournant le juge, que le gouvernement a entrepris d'édicter la règle ${ }^{23}$. Rien d'étonnant à cela : attaché à une lecture libérale du rapport de l'État au fait religieux, le juge - administratif, mais aussi judiciaire - n'a guère

20. SÉNAC R., L’invention de la diversité, Paris, PUF, 2012.

21. Sur cette réception des productions intellectuelles par les élites politiques, V. PORTIER PH., L'État et les religions en France, Une sociologie historique de la laïcité, Rennes, PUR, 2016.

22. Commaille J., Kaluszynski M. (dir.), La fonction politique de la justice, Paris, La Découverte, 2007.

23. Sur ce point, V. THÉRON S., « La substitution de la loi à la jurisprudence administrative : la jurisprudence codifiée ou remise en cause par la loi », RFDA 2004, p. 230. 
souhaité, au cours de ces dernières années, restreindre l'exercice de ce qu'il concevait comme une expression de la liberté de conscience ${ }^{24}$. On constatera cependant que cette stratégie de consolidation du politique s'est faite parfois avec le concours du juge, et notamment du Conseil constitutionnel comme il est arrivé en 2010.

Le juge administratif a eu à connaître du voile simple tout d'abord. À l'automne 1989, Lionel Jospin, ministre de l'Éducation, se trouve confronté, après que la loi d'orientation du 10 juillet 1989 a admis la liberté pour les collégiens et lycéens de manifester leurs opinions, à la situation problématique née du renvoi des trois jeunes filles voilées de Creil. Avec l'accord du Premier ministre, Michel Rocard, il décide de solliciter l'avis du Conseil d'État en vue de savoir si le port de signes religieux dans l'enclos scolaire est bien conforme au principe de laïcité. L'avis est remis le 27 novembre ${ }^{25}$. Fondée sur des textes de droit interne comme la loi du 28 mars 1882 sur l'enseignement primaire obligatoire, la Constitution de 1958, ou les lois de 1975 et de 1989 sur l'éducation, mais aussi sur des textes de droit international comme la Convention européenne des droits de l'homme de 1950 et les pactes relatifs aux droits civils et politiques, et aux droits sociaux, économiques et culturels de 1966, la position d'ouverture de la Haute Assemblée peut s'énoncer en un syllogisme. Majeure : la laiicité est, en effet, un principe fondateur de la République, et en son sein, de l'école publique. Le Conseil d'État cite, de ce point de vue, la loi Ferry de 1882, mais aussi la loi de Séparation de 1905 et les Constitutions de 1946 et 1958, qui ont, l'une et l'autre, fait de la laïcité une des quatre notes définitoires de la République. La mineure définit la laïcité. Elle se trouve rapportée à sa substance libérale : elle est la condition juridique de l'exercice de la liberté de conscience, et même, ce qui n'était pas nécessairement le cas sous Briand, du droit à la différence. De là vient la conclusion, qui laisse aux élèves une grande latitude quant à leur conduite vestimentaire, et qu'on retrouvera dans plusieurs arrêts subséquents comme l'arrêt Kherouaa de $1992^{26}$ : «Dans les établissements scolaires, le port par les élèves de signes par lesquels ils entendent manifester leur appartenance à une religion n'est pas par lui-même incompatible avec le principe de laiicité, dans la mesure où il constitue l'exercice de la liberté d'expression et de manifestation de croyances religieuses. " La règle est posée, qui admet cependant son exception : l'interdit est possible dans le cas notamment où l'affichage

24. Languille C., La possibilité du cosmopolitisme, Paris, Gallimard, 2015.

25. CE, avis, 27 nov. 1989, n 346.893.

26. CE, 2 nov. 1992, n 130394, Kherouaa et autres. 
identitaire induirait, du fait de son " caractère ostentatoire ou revendicatif ", une atteinte «à la liberté, à la dignité, à la sécurité de l'élève et des autres membres de la communauté éducative », " perturberait les activités d'enseignement $»$, «troublerait l'ordre dans l'établissement ${ }^{27}$. Dans sa circulaire du 12 décembre 1989, le ministre de l'Éducation, à une époque où une grande partie de la gauche cultive l'idée d'une « laïcité plurielle», reprend le dispositif du Conseil d'État : la liberté de l'élève est première, pourvu certes qu'elle ne le conduise pas à transgresser les limites à l'instant mentionnées.

La ligne libérale triomphe aussi au moment de l'affaire de la «burqa », vingt ans plus tard. Confronté aux hésitations juridiques de la mission parlementaire présidée par André Gerin, le Premier ministre, François Fillon, décide de consulter la juridiction de Palais-Royal sur le point de savoir s'il est possible de soumettre le port du voile intégral à un régime d'interdiction générale. Or, là encore, la juridiction administrative répond négativement. Dans son Étude relative aux possibilités juridiques d'interdiction du port du voile intégral en mars 2010, elle récuse trois arguments qui auraient pu fonder une loi de ce type. D'abord, l'argument de la laïcité. Celle-ci, rappelle le Conseil qui la saisit sous le concept de neutralité, ne concerne que les institutions publiques; elle ne s'applique pas en revanche dans les espaces de la société civile, pas plus qu'elle ne concerne les usagers du service public, sauf l'exception des établissements scolaires (que les juges du Palais-Royal ont finalement validée dans leur avis de janvier 2004 sur le projet de loi portant interdiction du port de signes religieux à l'école publique). Ensuite, l'argument de la dignité : le Conseil d'État déclare l'avoir déjà employé en la rapportant à une " exigence morale collective » ${ }^{28}$; il ajoute cependant que cet usage perfectionniste risque d'être récusé par la Cour européenne des droits de l'homme qui l'identifie volontiers pour sa part, de manière très libérale, à la possibilité de poser un libre choix. Enfin, l'argument de l'égalité : «Opposable à autrui, il n'a pas, explique le juge, vocation à être opposé à la personne elle-même, c'est-à-dire à l'exercice de sa liberté personnelle, laquelle peut,

27. Le texte exact est celui-ci : "Cette liberté ne saurait permettre aux élèves d'arborer des signes d'appartenance religieuse qui, par leur nature, par les conditions dans lesquelles ils seraient portés individuellement ou collectivement, ou par leur caractère ostentatoire ou revendicatif, constitueraient un acte de pression, de provocation, de prosélytisme ou de propagande, porteraient atteinte à la dignité ou à la liberté de l'élève ou d'autres membres de la communauté éducative, compromettraient leur santé ou leur sécurité, perturberaient le déroulement des activités d'enseignement et le rôle éducatif des enseignants, enfin troubleraient l'ordre dans l'établissement ou le fonctionnement normal du service public. »

28. CE, ass., 27 oct. 1995, n 136727, Commune de Morsang-sur-Orge, à propos de l'affaire du « lancer de nains». 
le cas échéant, la conduire à adopter un comportement susceptible d'être interprété comme consacrant son inégale situation. »Ces difficultés amènent l'autorité du Palais-Royal à déplacer l'espace de réflexion en s'interrogeant non plus sur le «port du voile intégral », mais sur la « dissimulation du visage dans l'espace public ». Elle aboutit à la même conclusion, en estimant que le motif de l'ordre public, seul susceptible d'être invoqué en l'espèce, ne pourrait en soi, même dans une définition élargie de son concept (intégrant, par exemple, les règles essentielles du vivre ensemble ou la « dignité de la personne »), entraîner l'approbation du Conseil constitutionnel, ni celle de la Cour européenne des droits de l'homme, en faveur d'une loi d'interdiction générale. Ne reste, à ses yeux, qu'une solution, sauf à remettre en cause le régime des libertés et à s'exposer, de ce fait, à la censure : une interdiction partielle de la dissimulation du visage dans des conditions particulières de temps et de lieu, en l'appuyant sur les requêtes de la sécurité publique et de la lutte contre la fraude. À travers cet avis, le Conseil d'État décrit l'état du droit ; il le prescrit aussi : comme l'avait relevé déjà la jurisprudence Baldy en 1917, la liberté, à ses yeux, doit être la règle, la restriction l'exception.

Dans les deux affaires, le pouvoir politique, dont les ténors déclarent de plus en plus volontiers que la souveraineté réside dans le peuple qu'ils représentent et non dans le juge, a décidé de forcer les choses, en entrant, contre l'opposition éventuelle de l'autorité judiciaire, dans un processus de transformation de la loi ${ }^{29}$. Encore fallait-il justifier l'opération. Deux stratégies ont été employées. Les gouvernants ont réquisitionné, d'une part, les moyens de la démocratie épistémique. L'État postmoderne, qui s'impose dans le monde occidental à partir des années 1970, a fait prévaloir le modèle d'Hermès sur celui de Jupiter ${ }^{30}$ : la loi ne peut plus s'imposer d'en haut sur un mode décisionniste; elle suppose un dialogue entre la société politique et la société civile, souvent façonné dans le dispositif des comités d'experts. Ce schéma de fonctionnement répond à une raison normative : dans un contexte marqué par la perte d'efficace de l'État légal-rationnel, il peut amener la loi à gagner en qualité ; mais aussi à une raison stratégique : il peut offrir à l'État de consolider la légitimité de ses décisions. La comitologie est au cœur de notre dossier. On le voit en 2004. La loi a été préparée par le travail de la

29. Galembert C. DE, «Forcer le droit à parler contre la burqa. Une judicial politics à la française? », RF sc. pol., n 64/4, 2014, p. 647-668. V. aussi le dossier qu'elle a dirigé sur le voile simple, "Le voile en procès ", Droit et société, n 68, 2008.

30. On emprunte cette distinction à François Ost, qui l'applique, quant à lui, au travail du juge. Ost F., «Jupiter, Hercule, Hermès : trois modèles du juge », in Bouretz P. (dir.), La force du droit, panorama des débats contemporains, Paris, Éd. Esprit, 1991, p. 241. 
commission sur l'application du principe de laïcité dans la République placée sous la présidence de Bernard Stasi, installée par le Président Jacques Chirac en juillet 2003. À l'instar de la commission Debré (sur le port des signes religieux à l'école) et de la mission Baroin (sur l'actualité du principe de la laïcité) qui travaillent au même moment en la rejoignant dans ses conclusions, celle-ci fera, après que les auditions l'ont persuadée des avancées du " communautarisme » au sein de l'école républicaine, cette préconisation que reprendra le législateur : «Dans le respect de la liberté de conscience et du caractère propre des établissements privés sous contrat, sont interdits dans les écoles, collèges et lycées les tenues et signes manifestant une appartenance religieuse ou politique. » La loi de 2010 s'inscrit de même dans le prolongement de la réflexion menée par un groupement ad hoc - la mission d'information sur la pratique du port du voile intégral sur le territoire national, installée en juillet 2009 sous la présidence d'André Gerin : s'il est composé exclusivement de parlementaires, lui aussi organise son travail, comme la commission Stasi, sur le fondement d'une série d'entretiens avec les représentants de la société civile (intellectuels, personnalités religieuses, leaders de groupements convictionnels, et, "même », une femme voilée). Le processus, ici également, aboutit à fermer le champ des possibles : analysant le voile intégral comme une "pratique radicale entre archaïsme culturel et prosélytisme intégriste », en tout « contraire aux valeurs de la République », la mission Gerin, en conclusion de son rapport, demande au pouvoir de proscrire le voile intégral pour les usagers des services publics, en raison du trouble qu'il fait courir à l'ordre public, en faisant valoir de surcroît que la quasi-totalité de ses membres souhaitait même son interdiction totale dans l'espace public. Certains observateurs ont relevé que cette expertise n'avait guère en fait, à rebours par exemple de celle menée au Québec par la commission Bouchard-Taylor sur les accommodements raisonnables en $2008^{31}$, répondu aux exigences de l'« agir communicationnel ». Tout aurait été conçu - la composition des deux instances, le choix de leurs rapporteurs, la sélection des personnes auditionnées, la conduite des débats, le recours aux exemples étrangers ${ }^{32}$ - pour faire de ces deux structures, la commission Stasi, la mission Gerin, les lieux d'un « agir stratégique », tournés, non point vers la définition objective du problème et la production argumentée

31. Même si celle-ci a fait aussi, du côté en particulier des nationalistes québécois comme Mathieu Bock-Côté, l'objet de critiques, moins sur son mode de fonctionnement, il est vrai, que sur ses conclusions.

32. BAubérot J., « La Commission Stasi vue par l'un de ses membres », French Politics, Culture E Society, $\mathrm{n}^{\circ} 22$ (3), 2004, p. 135-141. 
de sa solution, mais vers la construction, prévue d'avance, d'un récit venant justifier la nécessité de l'interdit ${ }^{33}$.

Le gouvernement a utilisé, d'autre part, les ressources de la démocratie judiciaire. Alec Stone a bien montré comment l'État de droit, avec son feuilletage d'institutions juridictionnelles, avait un effet ambivalent sur la souveraineté du politique : s'il exerce une pression sur le pouvoir, qu'il peut soumettre à la censure, il peut contribuer aussi à renforcer la légitimité de ses productions normatives ${ }^{34}$. Il importe ici de distinguer les deux affaires. La première, celle du voile simple, a donné lieu à une opération d'évitement. Le pouvoir a pris soin, simplement, de s'assurer, en s'adossant à la déposition devant la commission Stasi de Jean-Paul Costa, alors vice-président de la Cour européenne des droits de l'homme, que la Cour de Strasbourg, malgré l'article 9 de la convention de 1950, très protecteur de la liberté religieuse, ne remettrait pas en cause la conventionnalité de la loi. Cette anticipation n'a d'ailleurs pas été démentie par les diverses décisions produites à la suite des exclusions prononcées dans les lycées et collèges (Aktas, Bayrac, Ghazal, Jasvir Singh et Ranjit Singh c/ France du 30 juin 2009) : toutes ont conclu à la conformité de la loi du 15 mars 2004, en faisant valoir, « eu égard à la marge d'appréciation qu'il convient de laisser à chaque État », le risque «pour les libertés et l'ordre public ${ }^{35}$ emporté, dans le cas de la France, par le port des signes religieux dans l'enclos scolaire. Dans le même temps, alors que la question prioritaire de constitutionnalité n'existait pas encore, le pouvoir a contourné le Conseil constitutionnel en produisant une loi de convergence, trouvant le soutien quasi-unanime de la droite et de la gauche. Rémy Libchaber a décrit de la sorte ce mécanisme prudentiel : «Si les craintes d'un contrôle n'ont jamais cessé d'être présentes, notamment dans les auditions de la commission [Stasi], il y fut rappelé que le Conseil constitutionnel ne pourrait intervenir que s'il était saisi, ce qui suggérait un possible consensus pour l'éviter. ${ }^{36} \mathrm{La}$ deuxième affaire, celle du voile intégral, a suscité, quant à elle, un dispositif

33. Rappelons aussi, à cet égard, que le rapport d'étape de la Commission nationale consultative des droits de l'homme sur La laïcité aujourd'hui a été totalement étouffé. Remis au Premier ministre le 15 décembre 2003, il considérait notamment, " qu'il serait contre-productif de grossir des phénomènes politico-religieux marginaux, que certains cherchent à exploiter, alors que la situation d'ensemble autorise en réalité un certain optimisme».

34. Stone Sweet A., "Constitutional courts and parliamentary democracy », West European Politics, vol. 25/1, 2002, p. 77.

35. Il s'agit là des conditions nécessaires à la limitation de la liberté religieuse, prévues par l'article 9 \& 2 de la convention de 1950.

36. Libchaber R., « À la croisée des interprétations : le voile et la loi », RDT civ. 2004, p. 162. 
d'intervention. Le gouvernement de droite s'est, motu proprio, tourné vers le Conseil constitutionnel pour obtenir son aval. Il a procédé de manière très solennelle en confiant la saisine de la Haute Juridiction aux présidents de l'Assemblée nationale et du Sénat, ce qui n'avait jamais été fait auparavant. Cette opération inédite relevait d'une double intention : au plan interne, empêcher l'usage de la QPC, au plan externe, peser sur la CEDH. Il ne s'agissait pas vraiment ici d'entraver le pouvoir de nuisance de la gauche : celle-ci, en effet, sans apporter son vote au projet gouvernemental (à l'exception de quelques francs-tireurs réunis autour de Manuel Valls), avait signifié son adhésion implicite, en soutenant le 11 mai 2010 une résolution parlementaire qui condamnait le voile intégral ${ }^{37}$. Cette judicial policy va être couronnée de succès. Impressionnés sans doute par la mobilisation de la classe politique, elle-même en phase avec une opinion publique massivement acquise à la cause, les juges de la rue Montpensier, qui du reste avaient démontré dans des arrêts antérieurs leur souci de placer l'impératif de la cohésion nationale au cœur de leur système normatif ${ }^{38}$, sont allés dans le sens du pouvoir. Leur décision du 7 octobre, qui reprend le contenu de l'exposé des motifs de la loi votée par le Parlement, note que le texte portant interdiction de la dissimulation du visage dans l'espace public est bien conforme à la Constitution : « [Étant donné] les objectifs qu'il s'est assignés et compte tenu de la nature de la peine instituée en cas de méconnaissance de la règle fixée par lui, le législateur a adopté des dispositions qui assurent, entre la sauvegarde de l'ordre public et la garantie des droits constitutionnellement protégés, une conciliation qui n'est pas manifestement disproportionnée. ${ }^{39}$

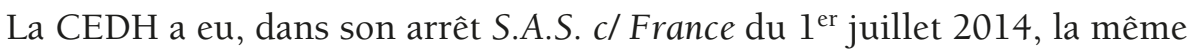
réaction : tout en assortissant sa décision de lourdes réserves (en raison des limites posées à la liberté religieuse), elle a admis la conventionnalité de la loi en reprenant l'idée, pointée par le législateur puis par le Conseil constitutionnel, selon laquelle la dissimulation du visage pouvait, en effet, porter atteinte à la sécurité publique certes, mais aussi « aux exigences minimales de la vie en société » que peut justifier « un choix de société ».

37. La résolution déclare : "L'Assemblée nationale considère que les pratiques radicales attentatoires à la dignité et à l'égalité entre les hommes et les femmes, parmi lesquelles le port d'un voile intégral, sont contraires aux valeurs de la République »: Résolution sur l'attachement au respect des valeurs républicaines face au développement de pratiques radicales qui y portent atteinte, adoptée le 11 mai 2010, TA n 459.

38. Cons. const., déc. 19 nov. 2004, n 2004-505 DC, Traité établissant une Constitution pour l'Europe.

39. Cons. const., déc. 7 oct. 2010, n 210-613 DC, Loi interdisant la dissimulation du visage dans l'espace public. 


\section{LES LÉGISLATIONS}

Sur le fondement de cette stratégie procédurale, par laquelle le pouvoir joue entre les différents niveaux de millefeuille étatique, s'est construite une autre normativité. On peut expliciter cette modification du droit en reprenant la distinction privé/public. Au début du $\mathrm{xx}^{\mathrm{e}}$ siècle, le législateur républicain avait pensé le régime des cultes en instituant, selon la logique libérale, une frontière hermétique entre les deux domaines. Les textes votés par le Parlement rendent plus indécises les limites : ensemble, sans qu'il y ait lieu de les opposer, ils soumettent, de manière inédite, le privé à la loi intrusive du public.

Examinons leur contenu, en premier lieu. La loi du 15 mars 2004 pose, à l'école publique, une interdiction générale. Il s'agit bien d'en finir avec la jurisprudence très prudente du Conseil d'État, que n'était pas parvenue à renverser la circulaire du 20 septembre 1994 signée par François Bayrou, alors ministre de l'Éducation. La règle nouvelle s'énonce en cette phrase, qui reprend les préconisations de la commission Stasi : «Dans les écoles, les collèges et les lycées publics, le port de signes ou tenues par lesquels les élèves manifestent ostensiblement une appartenance religieuse est interdit. Le règlement intérieur rappelle que la mise en œuvre d'une procédure disciplinaire est précédée d'un dialogue avec l'élève. » On notera l'évolution : ce sont les signes mêmes - ostensibles et non plus ostentatoires, ce qui entraîne à se placer du point de vue du récepteur et non plus de l'émetteur - qui sont visés, et non dorénavant les comportements. On voit là que s'inverse le jeu de la règle et de l'exception : comme le précisait le Conseil d'État dans une jurisprudence précitée (CE, 2 nov. 1992, Kherouaa), la loi hier autorisait les signes, sous réserve des conditions formulées plus haut; elle les interdit désormais, sauf à ce qu'ils soient « discrets » comme le précise la circulaire du 18 mai 2004. Résultante de toute une mobilisation intellectuelle, sociale, et politique, cette inversion se trouve justifiée, dans l'exposé des motifs de la loi, par une raison gestionnaire : il s'agit, en réponse aux revendications des chefs d'établissement, de résoudre par le haut les problèmes rencontrés en bas. Sans doute est-ce là la résultante de ce tropisme unitaire venu de la Révolution, qu'on n'éprouve pas de la même manière dans les pays anglosaxons. Mais la restriction doit aussi beaucoup à une raison axiologique : le législateur estime vouloir défendre tout à la fois l'unité de la République contre le risque "communautariste " et la liberté des jeunes filles contre les pressions de leur entourage, sans se poser la question de savoir si cette 
gestion étatique des corps ne met pas en péril l'autonomie de celles qui ont fait du port du voile un élément central de leur «mode de vie », ni interroger la croyance, qui est en fait au principe de la loi, d'une antinomie nécessaire entre le signe religieux et l'éthos démocratique. Cette centralité de la valeur, qui fait corps avec la centralité de l'école, apparaît notamment dans cette formule, extraite elle aussi de l'exposé des motifs de la loi :

« La réaffirmation du principe de laiicité à l'école, lieu privilégié d'acquisition et de transmission de nos valeurs communes, instrument par excellence d'enracinement de l'idée républicaine, paraît aujourd'hui indispensable. L'école doit en effet être préservée afin d'y assurer l'égalité des chances, l'égalité devant l'acquisition des valeurs et du savoir, l'égalité entre les filles et les garçons, la mixité de tous les enseignements, et notamment de l'éducation physique et sportive. »

Qu'en est-il de la loi du 11 octobre 2010 ? S'autorisant de la décision du Conseil constitutionnel rendue quatre jours plus tôt, elle édicte elle aussi un interdit général, cette fois destiné à l'espace public tout entier, mais sans qu'il soit question des signes religieux, en raison du risque de censure pour atteinte à la liberté de conscience. La proscription est formulée de la manière suivante, en ses articles 1 et $2:$ « Nul ne peut, dans l'espace public, porter une tenue destinée à dissimuler son visage. [...] L'espace public est constitué des voies publiques ainsi que des lieux ouverts au public ou affectés à un service public. » Comment justifier cette règle ? On remarquera que la laïcité n'est nullement invoquée ici. C'est autour de l'ordre public que s'articule le dispositif de la loi. Cette notion d'ordre public est un monument du droit français. Elle est, dans l'exposé des motifs de la loi, approchée de la manière suivante : « La défense de l'ordre public ne se limite pas à la préservation de la tranquillité, de la salubrité ou de la sécurité. Elle permet également de prohiber des comportements qui iraient directement à l'encontre de règles essentielles au contrat social républicain, qui fonde notre société. » On reviendra sur cette définition, qui doit beaucoup à l'intervention de Guy Carcassonne devant la mission Gerin. Retenons pour l'heure que si la dissimulation du visage - entendons en fait le voile intégral - introduit un trouble dans le code républicain des conduites, c'est parce qu'elle attente à trois de ses éléments essentiels. À la civilité, d'abord, qui a partie liée à ce que François Saint-Bonnet appelait, dans un article récent, la citoyenneté ${ }^{40}$. La République, explique le législateur, n'est pas un ensemble sérialisé, mais un ordre intégratif. Or, l'abstraction des visages

40. Saint-Bonnet. F. «La citoyenneté, fondement démocratique pour la loi anti-burqa », Jus Politicum, n ${ }^{\circ}$ 7, 2012. 
fait obstacle à la rencontre des citoyens : «La dissimulation systématique du visage dans l'espace public, contraire à l'idéal de fraternité, ne satisfait pas à l'exigence minimale de civilité nécessaire à la relation sociale. » On mentionne également la dignité. Sur ce point, le pouvoir politique se distancie, une nouvelle fois, du Conseil d'État qui en avait trouvé l'usage fragile juridiquement. Le Parlement ose l'imposer, en lui donnant une définition perfectionniste :

«Cette forme de réclusion publique, quand bien même elle serait volontaire ou acceptée, constitue à l'évidence, explique-t-il, une atteinte au respect de la dignité de la personne. Au reste, il ne s'agit pas seulement de la dignité de la personne ainsi recluse, mais également de celle des personnes qui partagent avec elle l'espace public et se voient traitées comme des personnes dont on doit se protéger par le refus de tout échange, même seulement visuel. »

Reste, enfin, l'égalité. Rien ne demeure là de la réflexion libérale, souvent portée par le juge européen ${ }^{41}$, qui considère que l'argument n'est pas opposable à la situation de ceux qui, de leur propre chef, optent pour un statut d'infériorité, rien non plus de la théorie de l'empowerment, qui estime que les femmes voilées peuvent construire leur idée de l'égalité indépendamment de la logique de la similitude homothétique à laquelle veut les soumettre le discours dominant : "Dans le cas du voile intégral, porté par les seules femmes, explique le législateur, cette atteinte à la dignité de la personne va de pair avec la manifestation publique d'un refus ostensible de l'égalité entre les hommes et les femmes, dont elle est la traduction. »

Les deux textes partagent-ils la même finalité ? Oui, sans doute. Dans leur conjugaison, ils contribuent à redéfinir, selon la même ligne d'inspiration, le schéma hexagonal de gestion de la croyance. Jusqu'aux années 1990, la laïcité française s'organise autour du principe de neutralité positive. La neutralité ? Toute une école a voulu, au début du $\mathrm{xx}^{\mathrm{e}}$ siècle, construire une politique restrictive : la fonction de l'État était, aux yeux de ses tenants, d'extraire les individus de la clôture des illusions religieuses. Maurice Allard, député socialiste du Var, était l'un des idéologues de cette « liberté de penser ». Le 10 avril 1905, lors du débat sur la loi de Séparation, il n'avait pas hésité à appeler ses collègues parlementaires à "poursuivre l'idée de la Convention et à achever l'œuvre de déchristianisation de la France ». Cette doctrine de l'État militant ne l'a pas emporté. La loi du 9 décembre, sous l'influence de Briand et de Jaurès, a institué, en fait, un État neutre, voué à protéger la croyance et l'incroyance

41. V. à cet égard la jurisprudence de la CEDH sur les expériences sadomasochistes. 
avec tout autant de force. Jean Rivero en rappelait ainsi le principe, dont il fait une condition de la liberté du sujet : « Le seuil du droit franchi, les disputes s'apaisent. Pour le juriste, la définition de la laïcité ne soulève pas de difficulté majeure. [...] Les textes législatifs, les rapports parlementaires qui les commentent, les circulaires qui ont accompagné leur mise en application ont toujours entendu la laïcité en un seul et même sens : celui de la neutralité religieuse de l'État». La positivité ? On peut saisir la neutralité sous le concept d'une abstention absolue de l'État à l'égard des univers de croyances : si le pouvoir politique ne bride pas, il ne soutient pas non plus. En réalité, malgré la séparation, l'État républicain a, très vite, en raison des résistances catholiques, manifesté son souci d'apporter son appui concret aux cultes, ou, plus exactement, aux individus croyants, au point qu'on a pu dire qu'il avait « transformé la liberté de religion en droit-créance ». Au plan financier, il a mis à disposition gratuite des assemblées religieuses les édifices du culte dont il était propriétaire, en assumant même, dans certains cas, les frais entraînés par leur réparation et leur entretien; il a également financé les aumôneries dans les lieux clos de l'espace étatique et, bientôt, subventionné massivement les écoles privées. Au plan symbolique, si l'État, pour marquer son équidistance à l'égard de toutes les familles d'opinion, exige de ses fonctionnaires une parfaite discrétion religieuse, il n'a nullement, pour autant, privé ses administrés de la possibilité de faire valoir leurs affiliations religieuses dans les services publics, ni même d'ailleurs dans les écoles publiques, comme le montrent la doctrine Jospin de 1989 mais aussi, bien que son interprétation puisse, sur ce point, porter à controverse, la lettre de Jules Ferry aux instituteurs en $1883^{42}$.

Initialement donc, la sphère étatique devait être absolument immune de toute influence religieuse; point, en revanche, la sphère sociale, qui devait échapper à toute neutralisation, en dehors des restrictions que pouvait justifier la préservation de l'ordre public matériel. Or, les deux lois sur le voile introduisent un coin dans cet ensemble recognitif. Elles ont, d'une part, élargi l'espace de la neutralité. La loi de 2004 a transformé l'espace scolaire : l'exhibition du signe y est devenue l'exception, comme si la neutralité devait s'étendre désormais jusqu'aux usagers du service public. En 2010, lorsqu'il s'est agi d'interdire le voile intégral, ont été visés pareillement les espaces intermédiaires - désignés sous le concept inédit d'« espace public »- tels que la voie publique, que Briand avait pris soin de laisser à la liberté ${ }^{43}$, ou les

42. V. sur tous ces points, PORTIER Ph., L'État et les religions en France, op. cit.

43. Comme on l'avait vu en 1905 lors de la discussion de l'amendement du député radicalsocialiste Charles Chabert, qui voulait interdire le port du costume religieux sur la voie publique. 
commerces. Il y a peu, à la faveur de l'effervescence provoquée par l'affaire Baby Loup, plusieurs parlementaires ont proposé de neutraliser totalement les espaces d'entreprise, en interdisant en leur sein tous les signes religieux, et non pas seulement le voile intégral. Les deux lois ont, d'autre part, substantialisé la signification de la neutralité. À l'origine, la règle, applicable aux seuls appareils d'État, avait pour fonction de permettre aux individus d'exercer leur liberté de croyance ou de conviction à l'abri de la domination des pouvoirs publics : elle était un instrument de protection des droits. Concernant également, désormais, le sujet ordinaire, elle supporte en fait une opération de rectification morale : on la comprend comme un instrument de diffusion des valeurs. Les choses sont annoncées déjà dans la loi de 2004. Comme on l'a vu plus haut, son exposé des motifs insiste, à l'instar du rapport Stasi, non point seulement sur la nécessité de placer les élèves à l'abri des pressions de leur entourage (ce qu'un libéral peut accepter, bien qu'il préfère les sanctions a posteriori, prises sur le fondement d'une enquête circonstanciée), mais sur la nécessité de les protéger contre des principes qui les fixeraient dans une forme d'aliénation idéologique (ce qu'un libéral ne peut que récuser, dans la mesure où, de son point de vue, chacun doit pouvoir choisir sa propre vie tant qu'il ne met pas en cause la liberté d'autrui). La loi de 2010 exprime la même idée, quoique de manière plus théorique, en travaillant le concept d'ordre public. On l'envisageait comme un ordre matériel hier. Sans récuser cette approche qui le relie aux notions de sécurité, de salubrité et de tranquillité, le législateur lui adjoint dorénavant une composante immatérielle, en la renvoyant aux «conditions minimales de la vie en commun ». On pourrait dire, au fond, qu'à l'épure kantienne de la Moralität, assise sur la coexistence des droits subjectifs ${ }^{44}$, se substitue la logique hégélienne de la Sittlichkeit, qui définit « le peuple libre [comme celui] dans lequel c'est la coutume (Sitte) qui constitue la substance de tous, dont tous et chacun savent individuellement l'effectivité et l'existence comme leur volonté et action $»^{45}$.

Au terme de ce parcours, on peut sans doute réévaluer les interprétations qui ont été données de la production législative de ces dernières années. Inscrite dans une perspective de longue durée, la thèse de Martha Nussbaum, typique d'une approche anglo-saxonne de la laïcité française, insiste sur la prégnance des structures culturelles : la Révolution française a établi un éthos

44. Bien que Kant incorpore, dans son modèle moral, l'idée de dignité.

45. Hegel G. W. F., Phénoménologie de l'esprit, trad. par J.-P. Lefebvre, Paris, Aubier, 1991, p. 460. 
de l'irréligion; les politiques du voile n'ont fait que persévérer dans cet être historique, selon une logique accentuée par le fait que le religieux dont il s'agit ici est l'islam, auquel la France se trouve liée par un passé compliqué et un présent parfois tragique. On lui a objecté ici que le régime des cultes s'était construit, sous la Troisième République, quoique dans le débat entre des positions très opposées, sur l'assise d'une philosophie libérale, accordant à l'expression du fait religieux une reconnaissance bien plus appuyée qu'on ne le dit ordinairement. Inspectant la toute dernière décennie, la thèse de Patrick Weil insiste, quant à elle, sur l'impact des ruptures politiques. La loi chiraquienne, votée par le Parti socialiste, demeurerait dans l'ordre du libéralisme, tandis que la loi sarkozyste, soutenue par la seule droite (ou presque), ferait signe vers un tournant autoritaire. Sans doute faut-il aussi nuancer cette approche, en rappelant que les deux lois ont été portées par un référentiel similaire de la restriction, né de l'urgence, repérable depuis les années 1990, de redonner du corps à une société du doute. L'interprétation qu'on a voulu soutenir décrit, en somme, une substitution de modèle. L'ordre libéral, dominant hier, était porté par la confiance dans la liberté du sujet. Le réaménagement en cours du régime de laïcité illustre l'entrée de la société contemporaine dans un néo-libéralisme qui, inversant la hiérarchie établie depuis Locke entre sécurité et autonomie, instaure le contrôle en principe même de sa rationalité politique. 